\title{
Electronic and magnetic properties of the ferromagnetic superconductor UCoGe
}

\author{
V.N. Antonov \\ Institute for Metal Physics, 36 Vernadsky Str., 03142 Kiev, Ukraine \\ E-mail: antonov@imp.kiev.ua
}

Received June 7, 2016, published online November 25, 2016

\begin{abstract}
The electronic structure and x-ray magnetic circular dichroism (XMCD) spectra of the ferromagnetic superconductor UCoGe at the $\mathrm{U} N_{4,5}$, Ge and Co $K$ and Co $L_{2,3}$ edges were investigated theoretically from first principles, using the fully relativistic Dirac linear muffin-tin orbital band structure method. The electronic structure is obtained with the local spin-density approximation (LSDA), as well as with a generalization of the LSDA $+U$ method which takes into account the non-diagonal occupation matrix (in spin indexes) of localized electrons. A stable ferromagnetic ground state was found. The uranium total magnetic moment is quite small (about $-0.171 \mu_{B}$ ) in the LSDA approximation as a result of almost complete cancellation between the spin magnetic moment of $0.657 \mu_{B}$ and the opposite orbital magnetic moment of $-0.828 \mu_{B}$, resulting from strong spin-orbit coupling at the uranium site. Valency of $U$ ion in UCoGe is close to $3+$. The ratio orbital and spin magnetic moments $M_{l} / M_{S}$ ranged from 1.163 in the GGA approach up to 2.456 for the LSDA+ $U$ calculations is smaller than the corresponding ratio for the free ion $\mathrm{U}^{3+}$ value (2.60), it can indicate a significant delocalization of the $5 f$-electron states due to the hybridization of the $\mathrm{U} 5 f$ electrons with the conduction band and Co $3 d$ electrons. The line shape of the dichroic spectra at the $U M_{5}$ and $M_{4}$ edges predicted by considering the magneto-optical selection rules as well as the occupation and the energy sequence of the $m_{j}$-projected partial densities of states. The theoretically calculated XMCD spectra at the $\mathrm{U} M_{4,5}$, Ge and Co $K$ and Co $L_{2,3}$ edges are in good agreement with the experimentally measured spectra.
\end{abstract}

PACS: 71.28.+d Narrow-band systems; intermediate-valence solids; 75.30.Mb Valence fluctuation, Kondo lattice, and heavy-fermion phenomena.

Keywords: heavy fermion, $\mathrm{x}$-ray magnetic circular dichnoism spectra.

\section{Introduction}

Uranium compounds exhibit a rich variety of properties to a large extent because of the complex behavior of their $5 f$ electrons. The $5 f$ states in $\mathrm{U}$ are intermediate between the itinerant $3 d$ electrons in transition metals and the localized $f$ electrons in rare-earth compounds. The determination of the electronic structure of $U$ compounds is a challenging task because in many of them the width of the $5 f$ bands, their spin-orbit splitting, and the on-site Coulomb repulsion in the partially filled $5 f$ shell are of the same order of magnitude and should be taken into account on the same footing. Interest in uranium compounds has recently been renewed, especially after the discovery of such unusual effects as heavy-fermion superconductivity and the coexistence of superconductivity and magnetism.

The coexistence of ferromagnetism (FM) and superconductivity (SC) has been at the forefront of condensed matter research since a pioneering paper by Ginzburg [1].
The interplay between two long-range orderings FM and $\mathrm{SC}$ is a fascinating aspect in strongly correlated electron systems because generally SC does not favorably coexist with FM since the FM moment gives rise to an internal magnetic field, which breaks the pairing state. During the last three decades, however, the discovery of a number of magnetic superconductors has allowed for a better understanding of how magnetic order and superconductivity can coexist. It seems to be generally accepted that antiferromagnetism with local moments coming from rare-earth (RE) elements readily coexists with type-II superconductivity [2]. This is because superconductivity and magnetism are carried by different types of electrons; magnetism is connected with deeply seated $4 f$ electrons, while superconductivity is fundamentally related to the outermost electrons such as $s, p$, and $d$ electrons. In the case of a ferromagnetic superconductor the situation is more complex because internal fields are not canceled out in the 
range of a superconducting coherence length in contrast with an antiferromagnetic superconductor. A fascinating aspect of this class of compounds is the observation that, within superconducting regime, a wealth of ground states can occur. Although a myriad of experiments have been devoted to the characterization of these ground states, a comprehensive understanding of the ferromagnetic superconductor properties at low temperature is still lacking. The ferromagnetic superconductor ground-state properties are highly sensitive to impurities, chemical composition, and slight changes of external parameters. This sensitivity indicates that a subtle interplay between different interactions produces a richness of experimental phenomena.

The coexistence of FM and SC was first discovered in $\mathrm{UGe}_{2}$ [3] under pressure in 2000, almost two decades after the discovery of $\mathrm{SC}$ in $\mathrm{CeCu}_{2} \mathrm{Si}_{2}$. Soon afterward, the $\mathrm{SC}$ was found in the weak ferromagnet URhGe for the first time at ambient pressure [4]. Recently UCoGe with identical crystal structure of URhGe was found to be a ferromagnetic superconductor, as well [5]. In all of these compounds, $T_{S C}$ is lower than $T_{C}$, indicating that SC phase exists in the FM phase, which is contrary to the case such as $\mathrm{ErRh}_{4} \mathrm{~B}_{4}$ [6].

UCoGe belongs to the family of intermetallic UTX compounds (where $\mathrm{T}$ is a transition metal and $\mathrm{X}$ is $\mathrm{Si}$ or $\mathrm{Ge}$ ) [7], crystallizing in the orthorhombic TiNiSi-type structure. Originally, it was reported to have a paramagnetic ground state [7]. Magnetization measurements show that UCoGe is a weak ferromagnet with a Curie temperature $T_{C}=3 \mathrm{~K}$ and a tiny ordered moment $\mu=0.03 \mu_{B}$. Superconductivity is observed with a resistance transition temperature $T_{s c}=0.8 \mathrm{~K}$. Additional thermal-expansion and specific-heat measurements provide clear evidence of bulk magnetism and superconductivity [5]. The proximity to a ferromagnetic instability suggests the electrons forming triplet Cooper pairs and superconductivity mediated by ferromagnetic fluctuations [5]. Nonlinear field response of the Shubnikov-de Haas frequency in UCoGe was observed above $20 \mathrm{~T}$ [8] and a possible field-induced topological Fermi surface transition, also known as a Lifshitz transition, supported by thermopower [9], and magnetoresistivity measurements [8,10]. Besides URhGe [4], UCoGe is the second known example of a ferromagnetic superconductor in the TiNiSi-type family of compounds.

There has been considerable impetus to understand the electronic structure and magnetism in $5 f$ materials, including this series of superconducting ferromagnets, owing to the wide variety of ground-state properties exhibited. Theoretical models are required to explain the properties of interactions and fluctuations, and a consequence of this is the need of direct knowledge of the spin and orbital moments. The x-ray magnetic circular dichroism technique developed in recent years has evolved into a powerful magnetometry tool to separate orbital and spin contributions to element specific magnetic moments. X-ray mag- netic circular dichroism experiments consist of measuring the absorption of $\mathrm{x}$ rays with opposite (left and right) states of circular polarization. A unique situation can be formed where the spin orbit coupling is typically of a similar magnitude to the crystal field. The delicate balance between these can lead to different ground states in apparently similar compounds, depending on the degree of localization of the $5 f$ electrons. For U, Hund's rules, which describe a local moment system, can be used to obtain the ratio of the orbital moment $\left(\mathrm{U}_{l}\right)$ and the spin moment $\left(\mathrm{U}_{s}\right)$. In a free ion the ratio is given by $\mathrm{U}_{l} / \mathrm{U}_{s}=-3.29$ for $\mathrm{U}^{4+}$ and $\mathrm{U}_{l} / \mathrm{U}_{s}=$ $=-2.60$ for $\mathrm{U}^{3+}$, and values below these are then used to characterize the itinerancy of the $5 f$ electrons [11].

The strong interplay between magnetism and superconductivity is a common feature of the ferromagnetic superconductors. While the magnetism of $\mathrm{UGe}_{2}$ and $\mathrm{URhGe}$ is well established and understood, this is not the case for UCoGe where the respective contribution of $U$ and Co is still under debate. There is an urgent need for a detailed knowledge of the magnetism of UCoGe. Band structure calculations [12] and neutron experiments [11] have endeavored to explore the orbital and spin part of the ordered moment, but contradictory results were published. On one hand, theoretical calculations in the LSDA approach [12] predict a small uranium moment $\left(\sim 0.1 \mu_{B}\right)$ due to an almost cancellation of substantial orbital and spin moments, and unexpectedly a large cobalt moment $\left(0.2-0.5 \mu_{B}\right)$ either parallel or antiparallel to the $U$ moment. On the other hand, comparison of NMR data of UCoGe led to conclude that the ferromagnetism in UCoGe originates predominantly from U $5 f$ electrons at least at low field [13]. Surprisingly, polarized neutron diffraction experiments [14] show that, in an applied field of $3 \mathrm{~T}$, the small ordered moment is essentially carried by the $U$ atoms $\left(\sim 0.1 \mu_{B}\right)$, while at $12 \mathrm{~T}$ a substantial moment $\left(\sim 0.2 \mu_{B}\right)$ antiparallel to the $\mathrm{U}$ moment is induced at the Co site and a parallel magnetization is observed in the interstitial regions $\left(\sim 0.3 \mu_{B}\right)$.

This paper is concentrated on the theoretical investigation of x-ray absorption spectra (XAS) and x-ray magnetic circular dichroism (XMCD) in UCoGe. Study of the $5 f$ electron shell in uranium compounds is usually performed by tuning the energy of the x ray close to the $M_{4,5}$ edges of uranium (located at 3552 and $3728 \mathrm{eV}$, respectively) where electronic transitions between $3 d_{3 / 2,5 / 2}$ and $5 f_{5 / 2,7 / 2}$ states occur. Recently XMCD measurements have been successfully performed for UCoGe compound [15,16]. Taupin et al. [15] measured XAS and XMCD spectra at the $\mathrm{U} M_{4,5}$ and $\mathrm{Co} / \mathrm{Ga} K$ edges. The orbital $\left(-0.70 \mu_{B}\right)$ and spin $\left(0.30 \mu_{B}\right)$ moments of $\mathrm{U}$ at $2.1 \mathrm{~K}$ and $17 \mathrm{~T}$ have been determined. The $\mathrm{XMCD}$ at the $\mathrm{Co} / \mathrm{Ge} K$ edges reveal the presence of small Co $4 p$ and Ge $4 p$ orbital moments parallel to the macroscopic magnetization. In addition, the Co $3 d$ moment is estimated to be at most of the order of $-0.1 \mu_{B}$ at $17 \mathrm{~T}$. Butchers et al. [16] measured the XAS and XMCD signal of UCoGe at $1.5 \mathrm{~K}$ and $6 \mathrm{~T}$ at $\mathrm{Co} L_{2,3}$ edges. They estimated the same value of 
the spin magnetic moment at $U$ site equal to $\sim 0.30 \mu_{B}$, however, $\mathrm{U}$ orbital moment was found to be equal $-0.40 \mu_{B}$ almost as twice smaller than data from Taupin et al. [15]. Both the publications obtain rather small ratio $\mathrm{U}_{l} / \mathrm{U}_{s}(-2.3$ in Ref. 15 and -1.3 in Ref. 16) suggesting a significant delocalization of the $5 f$-electron states in UCoGe.

There are some features in common for all the uranium compounds investigated up to now including UCoGe. First, the dichroism at the $M_{4}$ edge is much larger, sometimes one order of magnitude larger, than at the $M_{5}$ one. Second, the dichroism at the $M_{4}$ edge has a single negative lobe that has no distinct structure, on the other hand, two lobes, a positive and a negative one, are observed at the $M_{5}$ edge. With the above as background, we have performed calculations to evaluate the XMCD properties for UCoGe. The comparison between experiment and theory provides insight into the nature of the $5 f$ electrons and offers some evaluation of the suitability of several electronic structure methods for treating $5 f$ electrons.

This paper is organized as follows. Section 2 presents a description of the crystal structure of UCoGe and the computational details. Section 3 is devoted to the electronic structure and XMCD properties of UCoGe calculated in the LSDA, GGA and LSDA $+U$ approximations. The magnetooptical (MO) and XMCD theoretical calculations are compared to the experimental measurements. Finally, the results are summarized in Sec. 4.

\section{Crystal structure and computational details}

\subsection{Crystal structure}

The crystal structure of UCoGe is orthorhombic TiNiSitype (Pnma, space group No 62). The crystal structure is shown in Fig. 1, with the cell parameters taken from Huy et al. [5], and the internal atomic positions from Canepa et al. [17]. These parameters are shown in Table 1. The U atom forms the zig-zag chain along $a$ axis with the distance of $d_{U-U}=3.477 \AA$, which is close to the so-called Hill limit associated with the direct overlap of $5 f$ wave functions [18]. Of the $U$ atoms substructure each $U$ atom has four $U$ neighbors, two along the chain, and the other two in contiguous chains. The four neighbors form an unsymmetrical tetrahedron around the central $\mathrm{U}$ atom. This situation is similar to $\mathrm{C}$ in diamond, that is, the $\mathrm{U}$ atoms form a distorted diamond structure. $U$ has six nearest Co neighbors: one at the $2.8420 \AA$ distance, two at the $2.9330 \AA$ distance, two at

Table 1. Wyckoff positions $(x, y, z)$ for UCoGe [17]. Lattice constants $a=6.845 \AA, b=4.206 \AA$ and $c=7.222 \AA$ [5]

\begin{tabular}{c|c|c|c|c}
\hline \hline Atom & Site & $x$ & $y$ & $z$ \\
\hline \hline $\mathrm{U}$ & $4 c$ & 0.0101 & 0.25 & 0.7075 \\
$\mathrm{Co}$ & $4 c$ & 0.2887 & 0.25 & 0.4172 \\
$\mathrm{Ge}$ & $4 c$ & 0.1967 & 0.25 & 0.0870 \\
\hline \hline
\end{tabular}

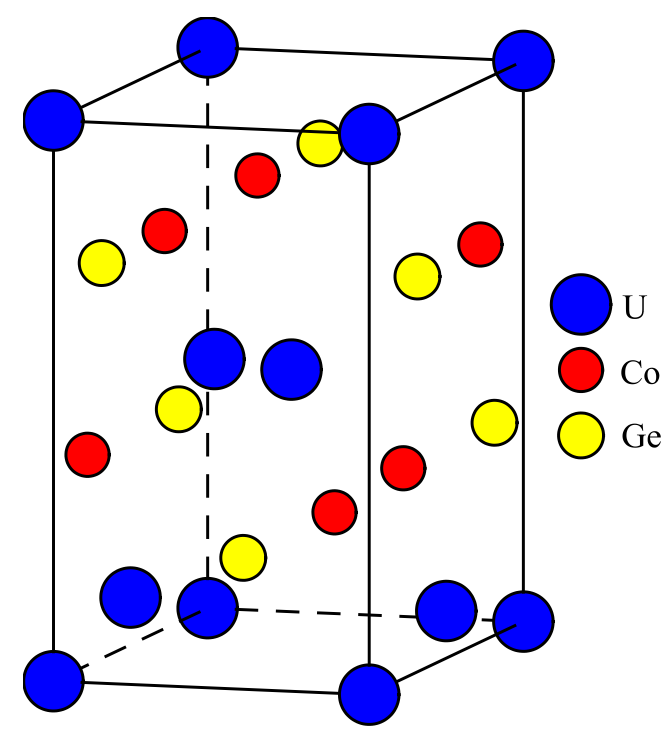

Fig. 1. (Color online) The crystal unit cell of UCoGe of the TiNiSi-type (Pnma, space group No 62) structure.

the $3.0715 \AA$ distance and one at the $3.1050 \AA$ distance. Two Ge atoms are situated at the $2.9378 \AA$ distance from $U$, one Ge is at the $3.0238 \AA$, another at the $3.0246 \AA$, and last two Ge atoms are at the $3.0358 \AA$ distance.

\subsection{X-ray magnetic circular dichroism}

Magneto-optical effects refer to various changes in the polarization state of light upon interaction with materials possessing a net magnetic moment, including rotation of the plane of linearly polarized light (Faraday, Kerr rotation), and the complementary differential absorption of left and right circularly polarized light (circular dichroism). In the near visible spectral range these effects result from excitation of electrons in the conduction band. Near x-ray absorption edges, or resonances, magneto-optical effects can be enhanced by transitions from well-defined atomic core levels to transition symmetry selected valence states.

Within the one-particle approximation, the absorption coefficient $\mu_{j}^{\lambda}(\omega)$ for incident $\mathrm{x}$ ray of polarization $\lambda$ and photon energy $\hbar \omega$ can be determined as the probability of electronic transitions from initial core states with the total angular momentum $j$ to final unoccupied Bloch states

$$
\begin{gathered}
\mu_{\lambda}^{j}(\omega)=\sum_{m_{j}} \sum_{n \mathbf{k}}\left|\left\langle\Psi_{n \mathbf{k}}\left|\Pi_{\lambda}\right| \Psi_{j m_{j}}\right\rangle\right|^{2} \delta\left(E_{n \mathbf{k}}-E_{j m_{j}}-\hbar \omega\right) \times \\
\times \theta\left(E_{n \mathbf{k}}-E_{F}\right),
\end{gathered}
$$

where $\Psi_{j m_{j}}$ and $E_{j m_{j}}$ are the wave function and the energy of a core state with the projection of the total angular momentum $m_{j} ; \Psi_{n \mathbf{k}}$ and $E_{n \mathbf{k}}$ are the wave function and the energy of a valence state in the $n$th band with the wave vector $\mathbf{k} ; E_{F}$ is the Fermi energy. 
$\Pi_{\lambda}$ is the electron-photon interaction operator in the dipole approximation

$$
\Pi_{\lambda}=-e \boldsymbol{\alpha} \mathbf{a}_{\lambda},
$$

where $\boldsymbol{\alpha}$ are the Dirac matrices, $\mathbf{a}_{\lambda}$ is the $\lambda$ polarization unit vector of the photon vector potential, with $a_{ \pm}=1 / \sqrt{2}(1, \pm i, 0), a_{\|}=(0,0,1)$. Here, + and - denotes, respectively, left and right circular photon polarizations with respect to the magnetization direction in the solid. Then, x-ray magnetic circular and linear dichroism are given by $\mu_{+}-\mu_{-}$and $\mu_{\|}-\left(\mu_{+}+\mu_{-}\right) / 2$, respectively. More detailed expressions of the matrix elements in electric dipole approximation may be found in Refs. 19-22. Matrix elements due to magnetic dipole and electric quadrupole corrections presents in Ref. 22.

Concurrent with the development of the x-ray magnetic circular dichroism experiment, some important magnetooptical sum rules have been derived [23-26].

For the $L_{2,3}$ edges the $l_{z}$ sum rule can be written as [21]

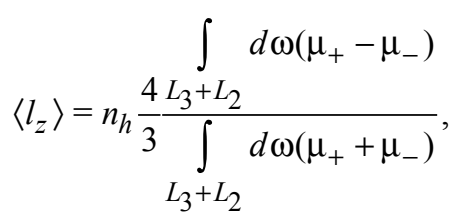

where $n_{h}$ is the number of holes in the $d$ band $n_{h}=10-n_{d},\left\langle l_{z}\right\rangle$ is the average of the magnetic quantum number of the orbital angular momentum. The integration is taken over the whole $2 p$ absorption region. The $s_{z}$ sum rule can be written as

$$
\left\langle s_{z}\right\rangle+\frac{7}{2}\left\langle t_{z}\right\rangle=n_{h} \frac{\int_{L_{3}} d \omega\left(\mu_{+}-\mu_{-}\right)-2 \iint_{L_{2}} d \omega\left(\mu_{+}-\mu_{-}\right)}{\int_{L_{3}+L_{2}} d \omega\left(\mu_{+}+\mu_{-}\right)},
$$

where $t_{z}$ is the $z$ component of the magnetic dipole operator $\mathbf{t}=\mathbf{s}-3 \mathbf{r}(\mathbf{r} \cdot \mathbf{s}) /|\mathbf{r}|^{2}$ which accounts for the asphericity of the spin moment. The integration $\int_{L_{3}}\left(\int_{L_{2}}\right)$ is taken only over the $2 p_{3 / 2}\left(2 p_{1 / 2}\right)$ absorption region.

In order to simplify the comparison of the theoretical x-ray isotropic absorption $M_{4,5}$ spectra of UCoGe to the experimental ones we take into account the background intensity which affects the high-energy part of the spectra. The shape of x-ray absorption caused by the transitions from inner levels to the continuum of unoccupied levels was first discussed by Richtmyer et al. in the early thirties [27]. The absorption coefficient with the assumption of equally distributed empty continuum levels is

$$
\mu(\omega)=\frac{C \Gamma_{c}}{2 \pi} \int_{E_{c f_{0}}}^{\infty} \frac{d E_{c f}}{\left(\Gamma_{c} / 2\right)^{2}+\left(\hbar \omega-E_{c f}\right)^{2}}
$$

where $E_{c f}=E_{c}-E_{f}, E_{c}$ and $\Gamma_{c}$ are the energy and the width of a core level, $E_{f}$ is the energy of empty continuum level, $E_{f_{0}}$ is the energy of the lowest continuum level, and $C$ is a normalization constant which has been used as an adjustable parameter.

\subsection{Calculation details}

The details of the computational method are described in our previous papers [28,29], and here we only mention some aspects specific to the present calculations. The calculations presented in this work were performed using the spinpolarized fully relativistic linear-muffin-tin-orbital (LMTO) method [30-32] for the experimentally observed lattice constants [5]. The LSDA part of the calculations was based on the spin-density functional with the Perdew-Wang [33] of the exchange-correlation potential. The exchange-correlation functional of a GGA-type was also used in the version of Perdew, Burke and Ernzerhof [34,35]. The basis consisted of the $s, p$, and $d$ LMTO's for Co and Ge sites and the $s, p$, $d$, and $f$ LMTO's for $\mathbf{U}$ site. The $\mathbf{k}$-space integrations were performed with the improved tetrahedron method [36] and the self-consistent charge density was obtained with 175 irreducible k-points in UCoGe.

The x-ray absorption and dichroism spectra were calculated taking into account the exchange splitting of core levels. The finite lifetime of a core hole was accounted for by folding the spectra with a Lorentzian. The widths of core level spectra for $\mathrm{U}, \mathrm{Co}$, and $\mathrm{Ge}$ was taken from Ref. 37. The finite apparative resolution of the spectrometer was accounted for by a Gaussian of width $0.6 \mathrm{eV}$.

It is a well-known fact that the LSDA calculations fail to produce the correct value of the orbital moment of uranium compounds [38-42]. In the LSDA, the Kohn-Sham equation is described by a local potential which depends on the electron spin density. The orbital current, which is responsible for $\mathrm{M}_{l}$, is, however, not included into the equations. This means, that although $M_{S}$ is self-consistently determined in the LSDA, there is no framework to determine simultaneously $\mathrm{M}_{l}$ self-consistently.

Numerous attempts have been made to better estimate $\mathrm{M}_{l}$ in solids. They can be roughly classified into two categories. One is based on the so-called current density functional theory [43-45] which is intended to extend density functional theory to include the orbital current as an extra degree of freedom, which describes $\mathrm{M}_{l}$. Unfortunately an explicit form of the current density functional is at present unknown. The other category includes orbital polarization (OP) [39-42], self-interaction correction (SIC) [46], and $\mathrm{LSDA}+U[47,48]$ approaches, which provide a means to calculate $\mathrm{M}_{l}$ beyond the LSDA scheme. 
Solovyev et al. [47] argued that the key parameter responsible for the exchange-correlation enhancement of the orbital magnetic moments in solids is the "Hubbard $U$ " rather than the intra-atomic Hund's second rule coupling, being consistent with a more general concept of the orbital polarization. This leads to a unified rotationally invariant LSDA $+U$ prescription for the orbital magnetism.

We have adopted the LSDA $+U$ method $[48,49]$ as a different level of approximation to treat the electron-electron correlation. We used a generalization of the LSDA $+U$ method which takes into account spin-orbit coupling so that the occupation matrix of localized electrons becomes nondiagonal in spin indexes. This method is described in detail in our previous paper [48] including the procedure to calculate the screened Coulomb $U$ and exchange $J$ integrals, as well as the Slater integrals $F^{2}, F^{4}$, and $F^{6}$. We used $J=0.5 \mathrm{eV}$, $U=2.0 \mathrm{eV}$ and $U=0.5 \mathrm{eV}$ for the $\mathrm{U}$ site. In the last case of $U=J=0.5 \mathrm{eV}, U_{\text {eff }}=U-J=0$ and the effect of the LSDA $+U$ comes from non-spherical terms which are determined by $F^{2}$ and $F^{4}$ Slater integrals. This approach is similar to the orbital polarization corrections mentioned above [21]. For the last LSDA $+U$ approach with $U=J=0.5 \mathrm{eV}$, $U_{\text {eff }}=U-J=0$ we will use the notation LSDA+OP through the paper.

\section{Results and discussion}

\subsection{Band structure}

For electronic structure calculations where there are heavy elements it is important to include spin-orbit interactions, and the magnetization is now dependent of the crystallographic direction. The results show that the most stable magnetization direction in $\mathrm{UCoGe}$ is in the $c$ axis, by 1.5 and $2.8 \mathrm{meV} /$ f.u. with respect to the $a$ and $b$ directions, respectively.

The fully relativistic spin-polarized LSDA partial DOSs of the ferromagnetic UCoGe compound are shown in Fig. 2. The results are in good agreement with previous band structure calculations [12,50-53]. The occupied part of the DOS can be decomposed into two regions. The first region, from -10.7 to $-7.9 \mathrm{eV}$, consists mainly of the Ge $4 s$ states, which are split off from the main valence-band group (see Fig. 2). There is a band gap from -7.9 to $-5.6 \mathrm{eV}$. The bottom of the main valence-band group is situated at $-5.6 \mathrm{eV}$. This band group represents mainly the Co $3 d$ states with the major part located below the Fermi level $\left(E_{F}\right)$ between -5.6 and $-0.07 \mathrm{eV}$. There is a pronounced hybridization between the Co $3 d$ and the $\mathrm{U} 5 f$ states between -5.7 and $2 \mathrm{eV}$. Typically for other UTM compounds, the spin-orbit coupling leads to two main $\mathrm{U} 5 f$ peaks, split into the $5 f_{5 / 2}$ and $5 f_{7 / 2}$ states (in energy range between -0.4 and $2.2 \mathrm{eV}$ ), shifted from each other by about $1 \mathrm{eV}$.

The bands crossing $E_{F}$ are dominated by the $\mathrm{U} 5 f_{5 / 2}$ states (see Fig. 3), being partly hybridized with the Co $3 d$ states, and these together create a metallic bond. The Co $3 d$

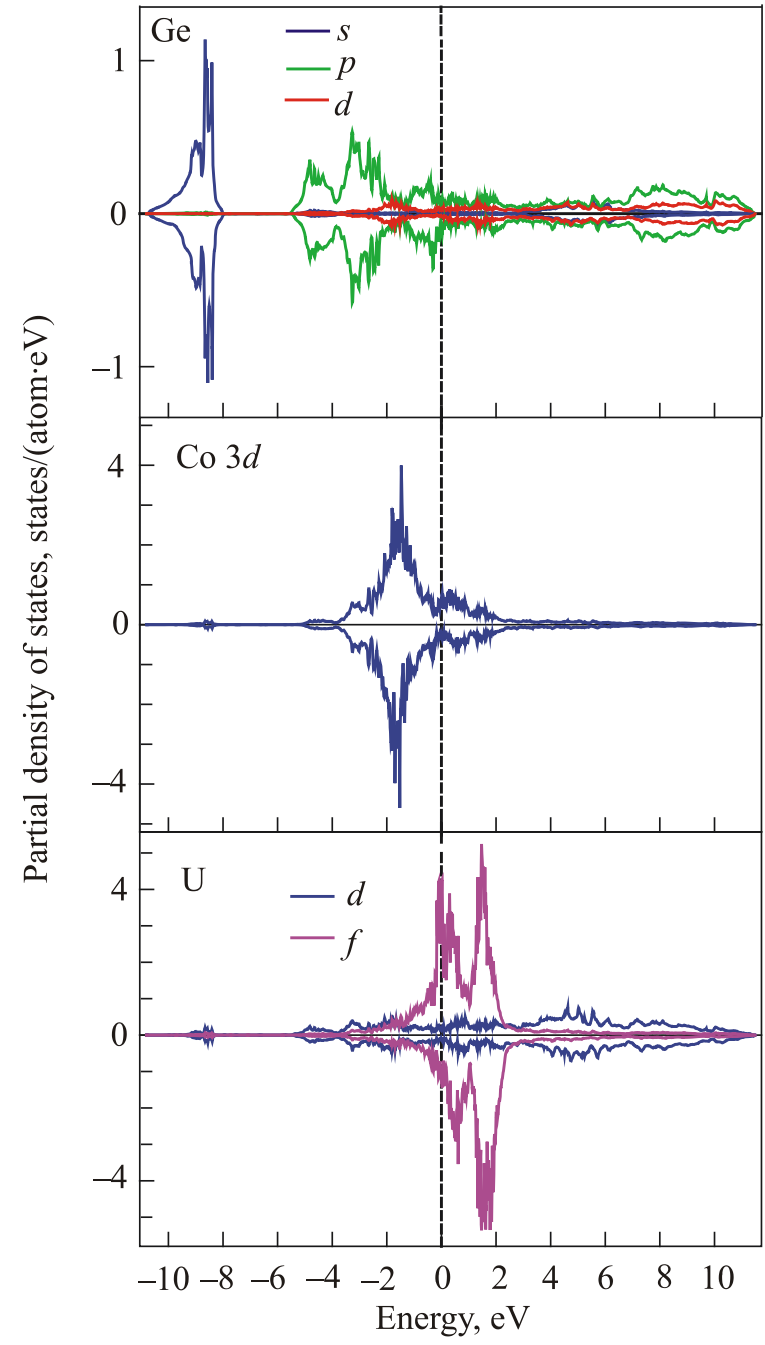

Fig. 2. (Color online) The LSDA self-consistent fully relativistic, spin-polarized partial DOSs (in states/(atom·eV)) of UCoGe.

states are clearly present in the whole range of valenceband energies with some tail at $E_{F}$. Some $\mathrm{U} 6 d$ and Ge $4 p$ band tails occur at $E_{F}$ as well, also contributing to a metallic bond. There is also a wide contribution from the $\mathrm{U} 6 d$ and Ge $4 p$ states in the same energy range as that of the U $5 f$ states, hybridizing with the latter.

Figure 4 shows ARPES spectra of UCoGe measured at $20 \mathrm{~K}$ with the photon energies equal to $500 \mathrm{eV}$ for $\mathrm{T}-\mathrm{Z}-\mathrm{T}$ high-symmetry line [53]. The experimental ARPES spectra are rather featureless. The spectra basically consist of three high-intensity parts located at around $E_{B}=E_{F}, 0.4 \mathrm{eV}$, and $0.8 \mathrm{eV}$. The former two are the contributions mainly from $\mathrm{U} 5 f$ states, while the last one is mainly from Co $3 d$ states although they are strongly hybridized (see Fig. 3). Furthermore, there are clear gaps in the intensity at around $E_{B}=$ $=0.2 \mathrm{eV}$ and between $0.5-0.7 \mathrm{eV}$. Figure 4 also shows the calculated band structures of UCoGe (full black curves) using the LSDA, LSDA $+\mathrm{OP}$, and $\mathrm{LSDA}+U$ approximations. Although, many dispersive bands exist in the calculation, and its comparison with the experimental spectra is not 


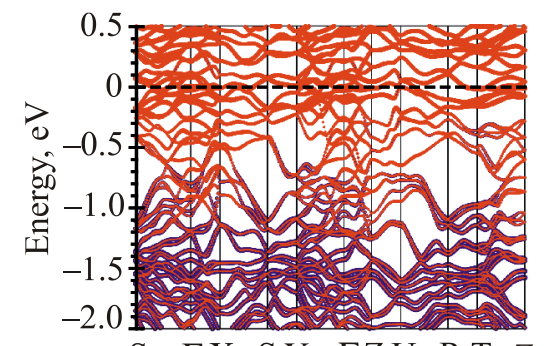

S $\Gamma \mathrm{X} \quad \mathrm{S} Y \quad \Gamma \mathrm{ZU}$ R T Z

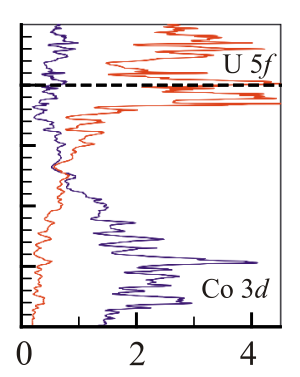

Fig. 3. (Color online) The LSDA self-consistent fully relativistic, spin-polarized energy band structure in "fat band" representation and $\mathrm{U} 5 f$ (red curve) and Co $3 d$ partial DOSs (in states/(atom·eV)) of UCoGe in the vicinity of $E_{F}$. The amounts of the $\mathrm{U} 5 f$ (open red circles) and Co $3 d$ (full blue circles) states characters are marked by the thickness of the bands.

straightforward the correspondences between the ARPES spectra and the calculations are clearly recognized. Meanwhile, the three approximations produce rather different structures of the energy bands in that energy interval. The LSDA approximation well describes the dispersion of U $5 f$ energy bands in close vicinity of the Fermi level, however, fails to deliver correct energy position of the bands around 0.4 and $0.8 \mathrm{eV}$. The LSDA+OP approximation, on the other hand, significantly improves the agreement between theoretically calculated and experimentally measured band positions in these two-energy intervals. Both the approximations well describe the gap in the intensity at around $E_{B}=0.2 \mathrm{eV}$ but not around $0.5-0.7 \mathrm{eV}$. For the LSDA $+U$ approach (with Hubbard $U=2 \mathrm{eV}$ ) the agreement between theory and ex-
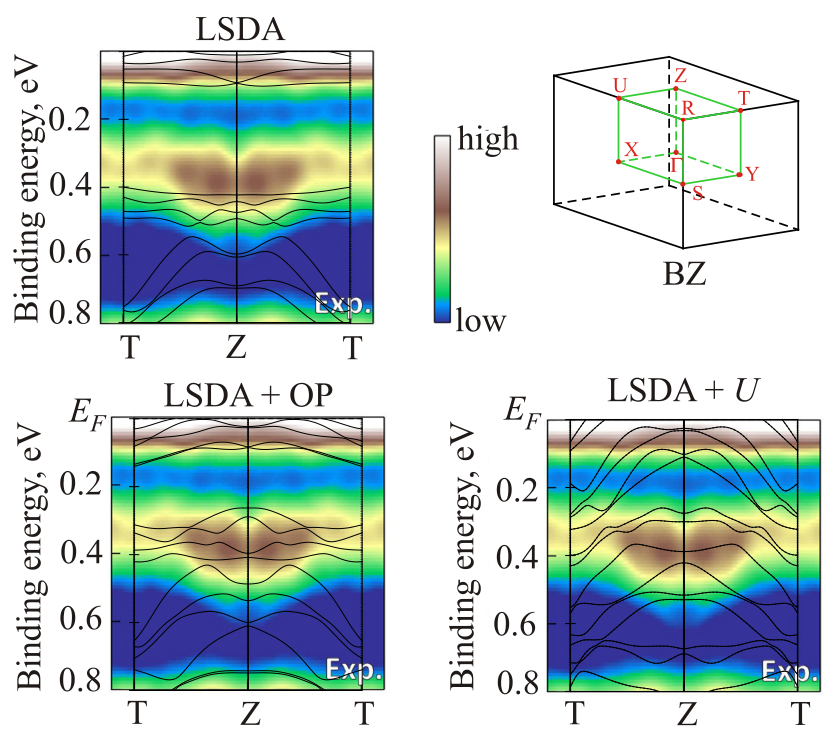

Fig. 4. (Color online) Blowup of the experimental ARPES spectra of UCoGe [53] and the band-structure calculations (full black curves) for the LSDA, LSDA+OP, and LSDA $+U$ approximations along the $\mathrm{T}-\mathrm{Z}-\mathrm{T}$ high-symmetry lines. periment becomes worse especially in states near $E_{F}$ and gap at $0.2 \mathrm{eV}$. We can conclude that the LSDA+OP approach produces the energy bands in closer agreement with the ARPES measurement in general comparing with other two approximations.

\subsection{XMCD spectra}

3.2.1. $U M_{4,5}$ spectra. In order to compare relative amplitudes of the $M_{4}$ and $M_{5}$ XMCD spectra we first normalize the corresponding isotropic x-ray absorption spectra (XAS) to the experimental ones taking into account the background scattering intensity as described in Sec. 2. Figure 5 shows the calculated isotropic $\mathrm{x}$-ray absorption and XMCD spectra in the LSDA, LSDA $+\mathrm{OP}$, and LSDA $+U$ approximations together with the experimental data [15]. The contribution from the background scattering is shown by dashed lines in the upper panel of Fig. 5.

The experimentally measured dichroic $M_{4}$ line consists of a simple nearly symmetric negative peak that has no distinct structure. Such a peak is characteristic of the $M_{4}$ edge of all uranium systems. The dichroic line at the $M_{5}$ edge has an asymmetric $s$ shape with two peaks - a stronger negative peak and a weaker positive peak. The dichroism at the $M_{4}$ edge is almost three times larger than at the $M_{5}$ one.

We recall that the $M_{4}\left(M_{5}\right)$ edge corresponds to $3 d_{3 / 2}\left(3 d_{5 / 2}\right) \rightarrow 5 f$ transitions. Because of the electric dipole selection rules $(\Delta l= \pm 1 ; \Delta j=0, \pm 1)$ the major contribution to the absorption at the $M_{4}$ edge stems from the transitions $3 d_{3 / 2} \rightarrow 5 f_{5 / 2}$ and that at the $M_{5}$ edge originates primarily from $3 d_{5 / 2} \rightarrow 5 f_{7 / 2}$ transitions, with a weaker contribution from $3 d_{5 / 2} \rightarrow 5 f_{5 / 2}$ transitions. For

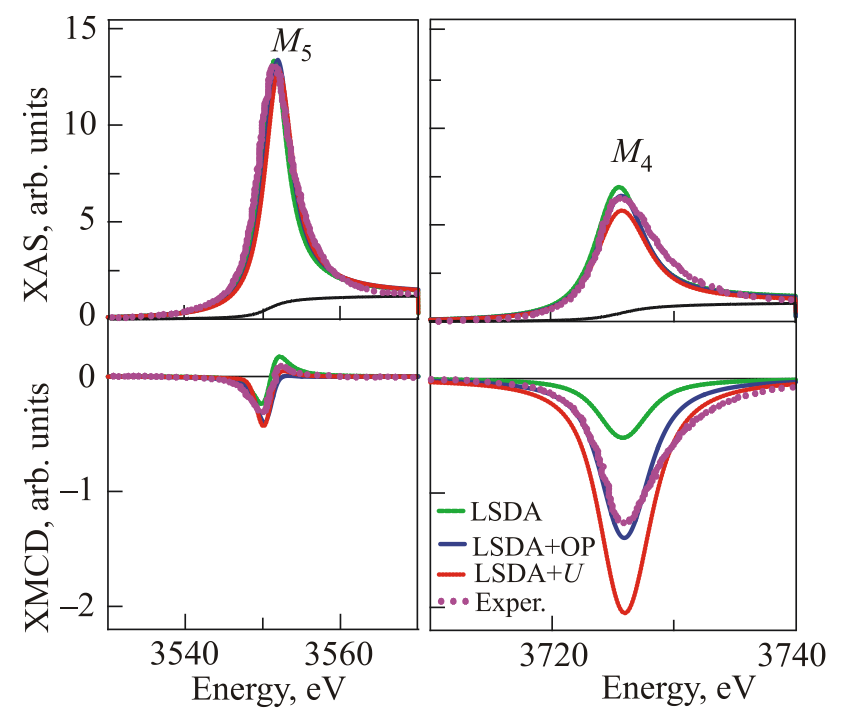

Fig. 5. (Color online) The experimental XAS and XMCD spectra [15] (open circles) at the U M4,5 edges in UCoGe compared with the theoretically calculated ones using the LSDA (dashed green curves), LSDA+OP (full blue curves), and LSDA $+U$ (dotted red curves) approximations. 
the later case the corresponding $3 d_{5 / 2} \rightarrow 5 f_{5 / 2}$ radial matrix elements are only slightly smaller than for the $3 d_{5 / 2} \rightarrow 5 f_{7 / 2}$ transitions. The angular matrix elements, however, strongly suppress the $3 d_{5 / 2} \rightarrow 5 f_{5 / 2}$ contribution. Therefore the contribution to XMCD spectrum at the $M_{5}$ edge from the transitions with $\Delta j=0$ is about 15 times smaller than the transitions with $\Delta j=1$.

The selection rules for the magnetic quantum number $m_{j} \quad\left(m_{j}\right.$ is restricted to $\left.-j, \ldots+j\right)$ are $\Delta m_{j}=+1$ for $\lambda=+1$ and $\Delta m_{j}=-1$ for $\lambda=-1$. Table 2 presents the dipole allowed transitions for x-ray absorption spectra at the $M_{5}$ and $M_{4}$ edges for left $(\lambda=+1)$ and right $(\lambda=-1)$ polarized $\mathrm{x}$ rays.

Table 2. The dipole allowed transitions from core $3 d_{3 / 2,5 / 2}$ levels to the unoccupied $5 f_{5 / 2,7 / 2}$ valence states for left $(\lambda=+1)$ and right $(\lambda=-1)$ polarized $\mathrm{x}$ rays

\begin{tabular}{c|c|c}
\hline \hline Edge & $\lambda=+1$ & $\lambda=-1$ \\
\hline \hline & $-5 / 2 \rightarrow-3 / 2$ & $-5 / 2 \rightarrow-7 / 2$ \\
& $-3 / 2 \rightarrow-1 / 2$ & $-3 / 2 \rightarrow-5 / 2$ \\
$M_{5}$ & $-1 / 2 \rightarrow+1 / 2$ & $-1 / 2 \rightarrow-3 / 2$ \\
& $+1 / 2 \rightarrow+3 / 2$ & $+1 / 2 \rightarrow-1 / 2$ \\
& $+3 / 2 \rightarrow+5 / 2$ & $+3 / 2 \rightarrow+1 / 2$ \\
& $+5 / 2 \rightarrow+7 / 2$ & $+5 / 2 \rightarrow+3 / 2$ \\
\hline \multirow{4}{*}{$M_{4}$} & $-3 / 2 \rightarrow-1 / 2$ & $-3 / 2 \rightarrow-5 / 2$ \\
& $-1 / 2 \rightarrow+1 / 2$ & $-1 / 2 \rightarrow-3 / 2$ \\
& $-5 / 2 \rightarrow-3 / 2$ & $+1 / 2 \rightarrow-1 / 2$ \\
& $+3 / 2 \rightarrow+5 / 2$ & $+3 / 2 \rightarrow+1 / 2$ \\
\hline \hline
\end{tabular}

To go further, we needs to discuss the characteristic of the $5 f$ empty DOS. Since $l$ and $s$ prefer to couple antiparallel for less than half-filled shells, the $j=l-s=5 / 2$ has a lower energy than the $j=l+s=7 / 2$ level. Due to the intra-atomic exchange interaction the lowest sublevel of the $j=5 / 2$ will be $m_{5 / 2}=-5 / 2$, however, for the $j=7 / 2$ the lowest sublevel will be $m_{7 / 2}=+7 / 2$. This reversal in the energy sequence arises from the gain in energy due to alignment of the spin with the exchange field [54].

The $5 f_{7 / 2}$ states are almost completely empty in all the uranium compounds. Therefore all the transitions listed in Table 2 are active in the $M_{5}$ absorption spectrum. The contribution from the first four transitions for $\lambda=+1$ cancels to a large extent with the contribution of the opposite sign from the last four transitions for $\lambda=-1$ having the same final states. Thus the XMCD spectrum of $\mathrm{U}$ at the $M_{5}$ edge $\left(I=\mu^{-}-\mu^{+}\right)$can be roughly approximated by the following sum of $m_{j}$-projected partial densities of states: $\left(N_{-7 / 2}^{7 / 2}+N_{-5 / 2}^{7 / 2}\right)-\left(N_{7 / 2}^{7 / 2}+N_{5 / 2}^{7 / 2}\right)$. Here we use the notation $N_{m}^{j}$ for the density of states with the total momentum $j$ and its projection $m_{j}$. As a result, the shape of the $M_{5}$ XMCD spectrum contains of two peaks of opposite signs - a negative peak at lower energy and a positive peak at higher energy. As the separation of the peaks is smaller than the typical lifetime broadening, the peaks cancel each other to a large extent, thus leading to a rather small signal. Since the splitting of states with $m_{j}= \pm\left|m_{j}\right|$ increases with the increase of the magnetization at the $\mathrm{U}$ site, the amplitude of the $M_{5}$ spectrum should be proportional to the U magnetic moment.

A rather different situation occurs in the case of the $M_{4}$ X-ray absorption spectrum. Usually in uranium compounds the $\mathrm{U}$ atom is in $5 f^{3}\left(U^{3+}\right)$ or $5 f^{2}\left(U^{4+}\right)$ configurations and has partly occupied $5 f_{5 / 2}$ states. In the first case the $5 f_{5 / 2}$ states with $m_{j}=-5 / 2,-3 / 2$, and $-1 / 2$ are usually occupied. The dipole allowed transitions for $\lambda=+1$ are $-1 / 2 \rightarrow+1 / 2,+1 / 2 \rightarrow+3 / 2$, and $+3 / 2 \rightarrow+5 / 2$ and those for $\lambda=-1$ are $+3 / 2 \rightarrow+1 / 2$. The transitions with the same final states $m_{j}=+1 / 2$ mostly cancel each other and the XMCD spectrum of $\mathrm{U}$ at the $M_{4}$ edge can be roughly represented by the sum $-\left(N_{3 / 2}^{5 / 2}+N_{5 / 2}^{5 / 2}\right)$. The corresponding analysis for the $5 f^{2}\left(U^{4+}\right)$ configuration with occupied $f_{5 / 2,-5 / 2}$ and $f_{5 / 2,-3 / 2}$ states shows that the dipole allowed transitions for $\lambda=+1$ are $-3 / 2 \rightarrow-1 / 2, \quad-1 / 2 \rightarrow+1 / 2$, $+1 / 2 \rightarrow+3 / 2$, and $+3 / 2 \rightarrow+5 / 2$ and for $\lambda=-1$ : $+1 / 2 \rightarrow-1 / 2$ and $+3 / 2 \rightarrow+1 / 2$. Again, the XMCD spectrum of $\mathrm{U}$ at the $M_{4}$ edge can be approximated by $-\left(N_{3 / 2}^{5 / 2}+N_{5 / 2}^{5 / 2}\right)$. This explains why the dichroic $M_{4}$ line in uranium compounds consists of a single nearly symmetric negative peak.

We should note, however, that the explanation of the XMCD line shape in terms of $m_{j}$-projected DOS's presented above should be considered as only qualitative. First, there is no full compensation between transitions with equal final states due to difference in the angular matrix elements; second, in our consideration we neglect cross terms in the transition matrix elements; third, there is no pure $5 f^{3}$ or $5 f^{2}$ configurations in uranium compounds. It is always difficult to estimate an appropriate atomic $5 f$ occupation number in band structure calculations. Such a determination is usually obtained by the integration of the $5 f$ electron charge density inside of the corresponding atomic sphere. In the particular UCoGe case, the occupation number of $\mathrm{U} 5 f$ states is around 2.92 in the LSDA calculations. We, however, should keep in mind that some amount of the $5 f$ states are derived from the so-called "tails" of Co $3 d$ and Ge $4 p$ states arising as a result of the decomposition of the wave function centered at $\mathrm{Co}$ and $\mathrm{Ge}$ atoms. The careful analysis in the case of $\mathrm{UPd}_{3}$ presented in Ref. 48 shows that the occupation number of the "tails" of $\mathrm{Pd} 4 d$ states sum up to give the $5 f$ occupation of 0.9 electrons in the $U$ atomic sphere. We should also note that due to the strong hybridization between $\mathrm{U} 5 f$ and Co $3 d$ states, the $\mathrm{U} 5 f_{7 / 2}$ states in $\mathrm{UCoGe}$ are not completely empty, some of them are occupied, also some amount of $\mathrm{U} 5 f_{5 / 2}$ states, which we have been considering as fully occupied, are partially empty. 
The overall shapes of the calculated and experimental uranium $M_{4,5}$ XMCD spectra correspond well to each other (Fig. 5). The major discrepancy between the calculated and experimental XMCD spectra is the size of the $M_{4}$ XMCD peak. The LSDA underestimates the integral intensity of the XMCD at the $M_{4}$ edge. As the integrated $\mathrm{XMCD}$ signal is proportional to the orbital moment [24] this discrepancy may be related to an underestimation of the orbital moment by LSDA-based computational methods (see Table 4). On the other hand, the LSDA $+U$ approximation gives larger intensity for the $M_{4} \mathrm{XMCD}$ spectrum in comparison with the experimentally measured one. It reflects the overestimation of the orbital moment at $\mathrm{U}$ site in the LSDA $+U$ calculations (Table 4). The LSDA+OP approximation, in contrast, gives good agreement in the amplitude of the negative peak at the $M_{4}$ edge. The LSDA+OP approximation also slightly better reproduces the shape of the $M_{5}$ XMCD spectrum in comparison with the LSDA and LSDA $+U$ approaches.

3.2.2. Co $L_{2,3}$ spectra. Figure 6 shows the calculated XAS and XMCD spectra at the Co $L_{2,3}$ edges in UCoGe together with the experimental spectra [16]. Because of the dipole selection rules, apart from the $4 s_{1 / 2}$ states (which have a small contribution to the XAS due to relatively small $2 p \rightarrow 4 s$ matrix elements) only $3 d_{3 / 2}$ states occur as final states for $L_{2}$ XAS for unpolarized radiation, whereas for the $L_{3}$ XAS the $3 d_{5 / 2}$ states also contribute [21]. Although the $2 p_{3 / 2} \rightarrow 3 d_{3 / 2}$ radial matrix elements are only slightly smaller than for the $2 p_{3 / 2} \rightarrow 3 d_{5 / 2}$ transitions the angular matrix elements strongly suppress the $2 p_{3 / 2} \rightarrow 3 d_{3 / 2}$ contribution [21]. Therefore neglecting the energy dependence of the radial matrix elements, the $L_{2}$ and the $L_{3}$ spectra can be viewed as a direct mapping of the DOS curve for $3 d_{3 / 2}$ and $3 d_{5 / 2}$ character, respectively. The Co $L_{3}$ XAS spectrum has a pronounced shoulder at the $L_{3}$ peak shifted by about $3 \mathrm{eV}$ with respect to the maximum to higher photon energy. The theory represents this shoulder but with smaller intensity in comparison with the experiment. Similar situation is observed for the Co $L_{2}$ spectrum where theory also underestimates the intensity of high energy shoulder.

Figure 6 (lower panel) shows the theoretically calculated Co $L_{2,3}$ XMCD spectra in UCoGe using the LSDA, LSDA+OP, and LSDA $+U$ approximations in comparison with the experimentally measured spectra [16]. A qualitative explanation of the XMCD spectra shape is provided by the analysis of the corresponding selection rules, orbital character and occupation numbers of individual $3 d$ orbitals. Table 3 presents the dipole allowed transitions for x-ray absorption spectra at the $L_{3}$ and $L_{2}$ edges for left and right polarized $\mathrm{x}$ rays.

The dichroism at the $L_{3}$ edge has a major negative peak with positive high energy shoulder. The LSDA calculations underestimate the intensity both the major negative peak and high energy shoulder. The LSDA $+O P$ and $\mathrm{LSDA}+U$ produce similar results, however, $\mathrm{LSDA}+\mathrm{OP}$
Table 3. The dipole allowed transitions from core $2 p_{1 / 2,3 / 2}$ levels to the unoccupied $3 d_{3 / 2,5 / 2}$ valence states for left $(\lambda=+1)$ and right $(\lambda=-1)$ polarized $\mathrm{x}$ rays

\begin{tabular}{c|c|c}
\hline \hline Edge & $\lambda=+1$ & $\lambda=-1$ \\
\hline \hline & $-3 / 2 \rightarrow-1 / 2$ & $-3 / 2 \rightarrow-5 / 2$ \\
$L_{3}$ & $-1 / 2 \rightarrow+1 / 2$ & $-1 / 2 \rightarrow-3 / 2$ \\
$2 p_{3 / 2} \rightarrow 3 d_{5 / 2}$ & $+1 / 2 \rightarrow+3 / 2$ & $+1 / 2 \rightarrow-1 / 2$ \\
& $+3 / 2 \rightarrow+5 / 2$ & $+3 / 2 \rightarrow+1 / 2$ \\
\hline & $-3 / 2 \rightarrow-1 / 2$ & $-1 / 2 \rightarrow-3 / 2$ \\
$L_{3}$ & $-1 / 2 \rightarrow+1 / 2$ & $+1 / 2 \rightarrow-1 / 2$ \\
$2 p_{3 / 2} \rightarrow 3 d_{3 / 2}$ & $+1 / 2 \rightarrow+3 / 2$ & $+3 / 2 \rightarrow+1 / 2$ \\
\hline$L_{2}$ & $-1 / 2 \rightarrow+1 / 2$ & $-1 / 2 \rightarrow-3 / 2$ \\
$2 p_{1 / 2} \rightarrow 3 d_{3 / 2}$ & $+1 / 2 \rightarrow+3 / 2$ & $+1 / 2 \rightarrow-1 / 2$ \\
\hline \hline
\end{tabular}

still better describes the relative intensities of the two fine structures.

3.2.3. Co and Ge K spectra. Figure 7 shows the calculated XAS and XMCD spectra at the Co $K$ edge using the LSDA, LSDA+OP, and LSDA $+U$ approximations in UCoGe together with the experimental spectra [15]. Because dipole allowed transitions dominate the absorption spectrum for unpolarized radiation, the absorption coefficient $\mu_{K}^{0}(E)$ (not shown) reflects primarily the DOS of unoccupied $4 p$-like states $N_{p}(E)$ of Co above the Fermi level. Due to the energy-dependent radial matrix element for the $1 s \rightarrow 4 p$ there is no strict one-to-one correspond-

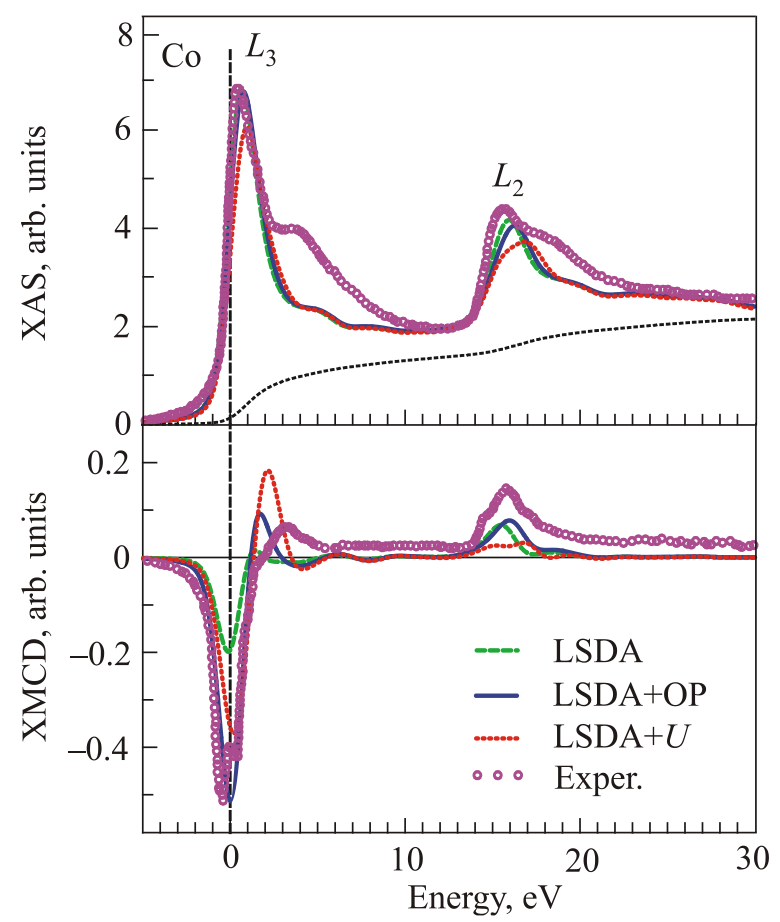

Fig. 6. (Color online) The experimental XAS and XMCD spectra [16] (open circles) at the Co $L_{2,3}$ edges in UCoGe compared with the theoretically calculated ones using the LSDA (dashed green curves), LSDA+OP (full blue curves), and LSDA $+U$ (dotted red curves) approximations. 


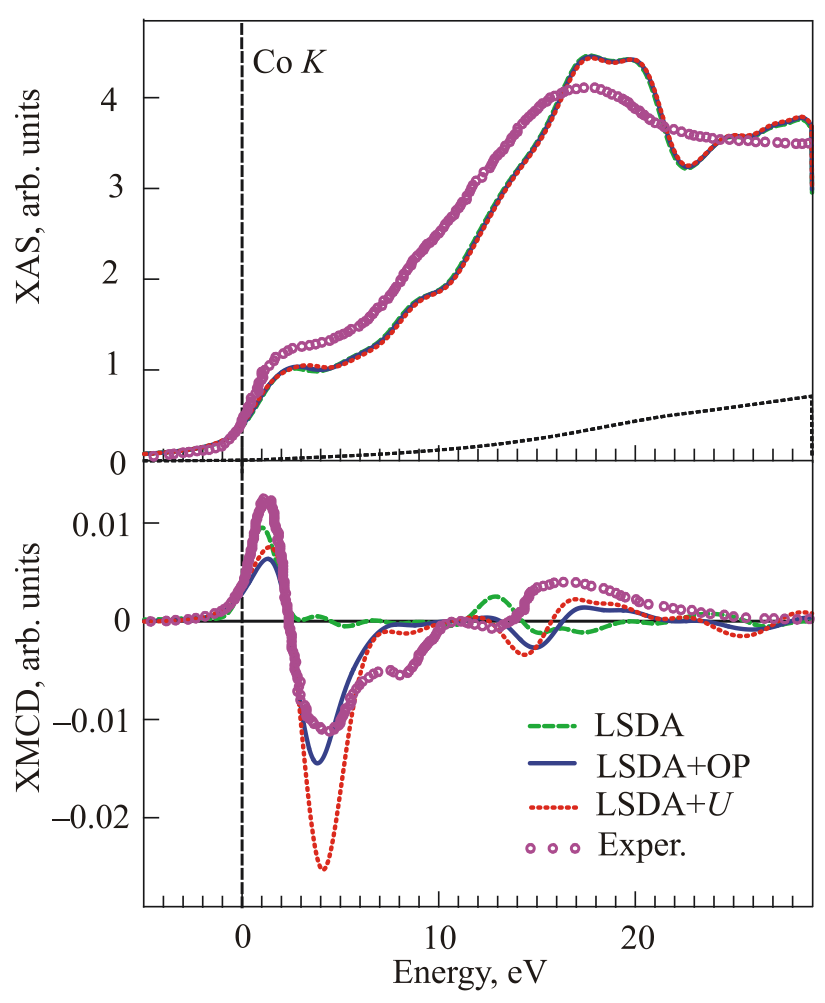

Fig. 7. (Color online) The experimental XAS and XMCD spectra [15] (open circles) at the Co $K$ edge in UCoGe compared with the theoretically calculated ones using the LSDA (dashed green curves), LSDA+OP (full blue curves), and LSDA $+U$ (dotted red curves) approximations.

ence between $\mu_{K}(E)$ and $N_{p}(E)$. The exchange splitting of the initial $1 s$-core state is extremely small [55] therefore only the exchange and spin-orbit splitting of the final $4 p$ states is responsible for the observed dichroism at the $K$ edge. For this reason the dichroism is found to be very small (Fig. 7). It was first pointed out by Gotsis and Strange [56] as well as Brooks and Johansson [57] that XMCD $K$-spectrum reflects the orbital polarization in differential form $d\left\langle l_{z}\right\rangle / d E$ of the $p$ states. It gives a rather simple and straightforward interpretation of the Co XMCD spectrum at the $K$ edge [58].

The x-ray absorption spectrum at the Co $K$ edge consists of the major peak at around $17 \mathrm{eV}$ above the edge and low energy shoulder at $2 \mathrm{eV}$. Theory well describe the energy position of these fine structures. All the three approximations produce rather similar $K$ XAS spectra. However, the deviations in the different approximations are well recognized for the XMCD spectra (lower panel of Fig. 7). The LSDA produces well the low-energy positive peak at $1 \mathrm{eV}$ but completely fails to describe the second negative peak at $4 \mathrm{eV}$ above the edge. The LSDA $+U$ calculations overestimate the intensity of this peak. The LSDA+OP gives the best agreement with experimental spectrum.

Figure 8 shows the calculated XAS and XMCD spectra at the Ge $K$ edge using the LSDA, LSDA+OP, and
LSDA $+U$ approximations in UCoGe together with the experimental spectra [15]. Theory well reproduces the major fine structures of Ge $K$ XAS spectrum: the main peak at $9 \mathrm{eV}$, low energy shoulder at $2 \mathrm{eV}$ and high energy shoulder at $13 \mathrm{eV}$. However, theory overestimates the intensity of last shoulder. Again, all the three approximations give identical XAS spectra.

In contrast, these approaches give very different dichroism spectra. The LSDA approach underestimates the dichroism at the Ge $K$ edge (lower panel of Fig. 8), the $\mathrm{LSDA}+U$ overestimates it and only the LSDA $+\mathrm{OP}$ give reasonable agreement with experimental measurements.

It is important to note that the Co and Ge $K$ XMCD spectra (Figs. 7 and 8) which reflect energy distribution of the $4 p$ valence state are much more sensitive to the type of the approximation for the U $5 f$ states in comparison with the corresponding Co $L_{2,3}$ XMCD spectra (Fig. 6). This fact indicates the stronger $\mathrm{U} 5 f-\mathrm{Co}(\mathrm{Ge}) 4 p$ hybridization in comparison with $\mathrm{U} 5 f$ - Co $3 d$ one due to more expanded character of the $4 p$ wave functions.

\subsection{Magnetic moments}

In magnets, the atomic spin $M_{s}$ and orbital $M_{l}$ magnetic moments are basic quantities and their separate determination is therefore important. Methods of their experimental determination include traditional gyromagnetic ratio meas-

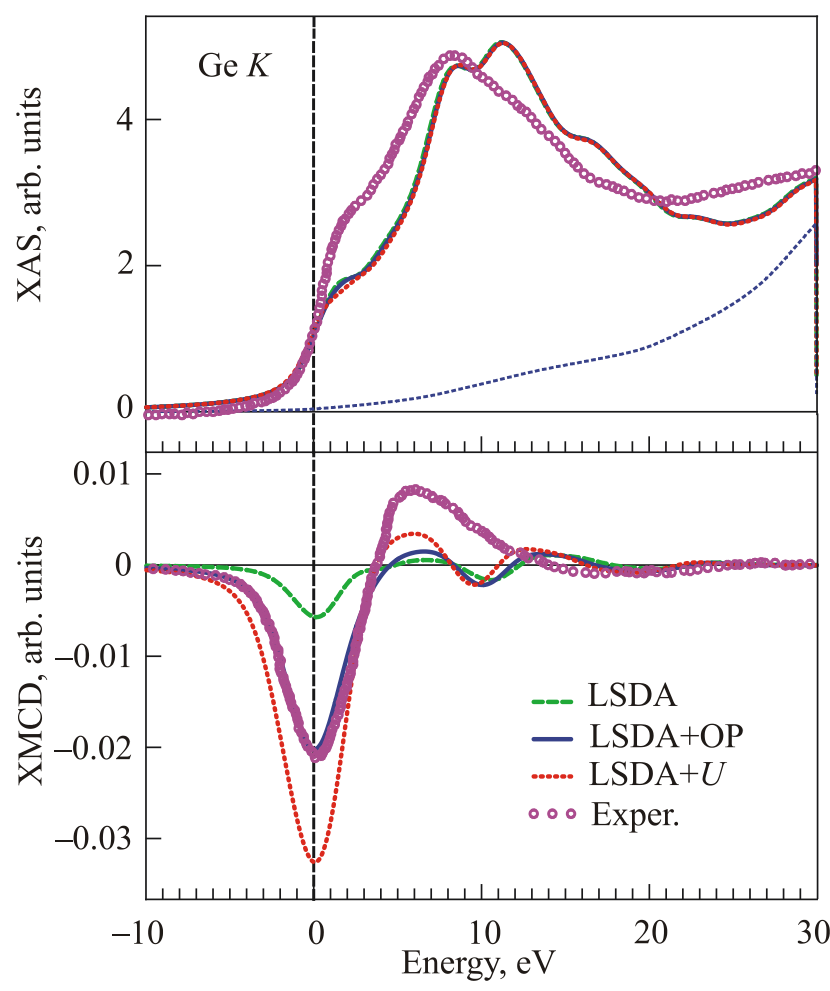

Fig. 8. (Color online) The experimental XAS and XMCD spectra [15] (open circles) at the Ge $K$ edge in UCoGe compared with the theoretically calculated ones using the LSDA (dashed green curves), LSDA+OP (full blue curves) and LSDA $+U$ (dotted red curves) approximations. 
urements [59], magnetic form factor measurements using the neutron scattering [60], and magnetic $\mathrm{x}$ ray scattering [61]. In addition to these, the recently developed $x$ ray magnetic circular dichroism combined with several sum rules [24,25] has attracted much attention as a method of site- and symmetry-selective determination of $M_{s}$ and $M_{l}$.

The experimentally observed net magnetization in UCoGe are highly controversial. On one hand, Huy et al. [5] estimated the total magnetic moment as quite small $\left(M_{t}=0.03 \mu_{B}\right.$ per f.u.), on the other hand, the effective paramagnetic moment found by Troc and Tran is much larger $M_{t}=1.7 \mu_{B}[62]$. The XMCD measurements provide total magnetic moments in between of these extreme values: $0.16 \mu_{B}$ [16] and $0.44 \mu_{B}$ [15]. Our band structure calculations produce the total magnetic moment equal to $0.298,0.411,1.345$, and $2.007 \mu_{B}$ for the LSDA, GGA, $\mathrm{LSDA}+\mathrm{OP}$, and LSDA $+U$ approximations, respectively.

Table 4 presents the comparison between calculated and experimental magnetic moments in UCoGe. It is clear that in the case of LSDA as well as the GGA calculations, the values of antiparallel spin and orbital moments on a uranium atom almost compensate each other. The spin and orbital moments on the Co atom are rather small, but parallel, and thus enhance each other. The total moment per $\mathrm{U}$ atom aligned along the $c, b$, and $a$ axes is as small as $-0.171,-0.123$, and $-0.164 \mu_{B}$, respectively, in the LSDA approach. At the same time, the Ge atom delivers a negligibly negative total magnetic moment. Finally, UCoGe has $M_{\text {tot }}$ (per f.u.) equal to about $-0.298,-0.246$, and $-0.333 \mu_{B}$, for alignments along the above three axes, respectively. The situation is changed when OP correction is included in the $\mathrm{U} 5 f$ states in the calculations. This leads to a marked increase of especially the orbital moment $M_{l}$ on the $\mathrm{U}$ site $\left(-2.093 \mu_{B}\right)$ and, hence, the value of the total $U$ magnetic moment, which becomes as large as $-1.073 \mu_{B}$ for the $c$ axis. A similar situation also occurs for the remaining axes. The LSDA $+U$ produces even larger $U$ orbital moment $\left(-3.077 \mu_{B}\right)$ and the total magnetic moment became equal to $-1.824 \mu_{B}$ at the $U$ site.
To investigate a possible error of the sum rules we compare the spin and orbital moments obtained from the theoretically calculated XAS and XMCD spectra through the sum rules with directly calculated LSDA and LSDA+OP values in order to avoid additional experimental problems. The sum rules reproduce the spin magnetic moments within $7 \%, 15 \%$, and $22 \%$ and the orbital moments within $5 \%, 21 \%$, and $0 \%$ for the $\mathrm{U}, \mathrm{Co}$, and $\mathrm{Ge}$ sites, respectively, for the LSDA approach (Table 4).

Taupin et al. [15] experimentally determined the branching ratio $B$ for the $3 d_{3 / 2,5 / 2} \rightarrow 5 f$ transition of $\mathrm{U}$ as $B=A_{5 / 2} /\left(A_{5 / 2}+A_{3 / 2}\right)$, where $A_{5 / 2}$ and $A_{3 / 2}$ are the integrated areas of the isotropic white lines at the $M_{5,4}$ edges, respectively. $B$ was found to be 0.701 . Thus the experimentally determined $B$ is in between those calculated for the $5 f^{2}\left(\mathrm{U}^{4+}\right)$ and $5 f^{3}\left(\mathrm{U}^{3+}\right)$ electronic configurations ( 0.686 and 0.729 , respectively) [15]. Thus the $5 f$ electron count is $2<n_{e}^{5 f}<3$. This is in agreement with our band structure calculations, $n_{e}^{5 f}$ is estimated to be 2.92 (in the LSDA calculations) and 2.96 (for the GGA approach), as well as with core level photoelectron spectroscopy [64] which shows that $n_{e}^{5 f}$ is less than but close to 3 . These results invalidate the occurrence of $\mathrm{U}^{4+}$ ions as suggested from neutron form factor analysis [14].

From the ratio $M_{l} / M_{S}$ the degree of localization of $5 f$ states can be estimated. If this ratio fall below the free ion $\mathrm{U}^{3+}$ value (2.60), it can indicate a significant delocalization of the $5 f$ electron states due to the hybridization of the $\mathrm{U} 5 f$ electrons with the conduction band and Co $3 d$ electrons [11]. This ratio is range from 1.163 in the GGA approach up to 2.456 for the $\mathrm{LSDA}+U$ calculations. The XMCD measurements estimate this ratio equal to 1.3 [16] and 2.34 [15]. Therefore, one can conclude that the on-site Coulomb repulsion on the $U$ site is suppressed, most likely because of the strong hybridization between $\mathrm{U} 5 f$ and Co $3 d / 4 p$ and Ge $4 p$ states. As a consequence, U $5 f$ electrons in UCoGe demonstrate almost purely itinerant behavior.

Table 4. The experimental and calculated spin $M_{s}$, orbital $M_{l}$, and total $M_{t}$ magnetic moments (in $\mu_{B}$ ) of UCoGe at $\mathrm{U}$, Co and Ge sites with magnetization along $c$ direction

\begin{tabular}{c|c|c|c|c|c|c|c|c|}
\hline \hline \multirow{2}{*}{ Method } & \multicolumn{3}{|c|}{$\mathrm{U}$} & \multicolumn{3}{c|}{ Co } & \multicolumn{2}{c}{$\mathrm{Ge}$} \\
\cline { 2 - 8 } & $M_{s}$ & $M_{l}$ & $M_{t}$ & $-M_{l} / M_{s}$ & $M_{s}$ & $M_{l}$ & $M_{s}$ & $M_{l}$ \\
\hline \hline LSDA & 0.657 & -0.828 & -0.171 & 1.453 & -0.100 & -0.019 & -0.009 & -0.001 \\
GGA & 0.955 & -1.105 & -0.150 & 1.163 & -0.201 & -0.041 & -0.017 & -0.002 \\
LSDA+OP & 1.020 & -2.093 & -1.073 & 2.052 & -0.191 & -0.066 & -0.017 & -0.002 \\
LSDA+U & 1.253 & -3.077 & -1.824 & 2.456 & -0.135 & -0.032 & -0.007 & -0.009 \\
LSDA [12] & 1.076 & -1.215 & -0.139 & 1.129 & -0.231 & -0.057 & -0.036 & -0.002 \\
LSDA+OP [12] & 1.254 & -2.666 & -1.412 & 2.126 & -0.300 & -0.183 & -0.045 & -0.010 \\
LSDA [63] & 1.083 & -1.181 & -0.098 & 1.090 & -0.472 & -0.063 & -0.026 & 0 \\
Exper. [15] & 0.297 & -0.695 & -0.398 & 2.34 & - & - & - & - \\
Exper. [16] & 0.30 & -0.40 & -0.16 & 1.3 & -0.16 & - & - & - \\
Sum rules (LSDA) & 0.609 & -0.868 & -0.259 & 1.425 & -0.115 & -0.015 & -0.007 & -0.001 \\
\hline \hline
\end{tabular}




\section{Summary}

We have studied by means of an $a b$ initio fullyrelativistic spin-polarized Dirac linear muffin-tin orbital (LMTO) method the electronic structure and the x-ray magnetic circular dichroism in UCoGe at the $\mathrm{U} M_{4,5}$, Co $L_{2,3}$, Co $K$, and Ge $K$ edges.

The bands crossing $E_{F}$ are dominated by the $\mathrm{U} 5 f_{5 / 2}$ states, being partly hybridized with the Co $3 d$ states, and these together create a metallic bond. We found that the LSDA+OP approach produces the energy bands in closer agreement with the ARPES measurement in general comparing with the LSDA and LSDA $+U$ approximations. In the case of LSDA as well as the GGA calculations, the values of antiparallel spin and orbital moments on a uranium atom almost compensate each other. The spin and orbital moments on the Co atom are rather small, but parallel, and thus enhance each other. The total moment per $\mathrm{U}$ atom aligned along the $c, b$, and $a$ axes is as small as $-0.171,-0.123$, and $-0.164 \mu_{B}$, respectively. The situation is changed when OP correction is included in the $\mathrm{U} 5 f$ states in the calculations. This leads to a marked increase of especially the orbital moment $M_{l}$ on the $\mathrm{U}$ site $\left(-2.093 \mu_{B}\right)$ and, hence, the total $\mathrm{U}$ magnetic moment, which becomes as large as $-1.073 \mu_{B}$ for the $c$ axis. A similar situation also occurs for the remaining axes.

The experimentally measured dichroic $M_{4}$ line consists of a simple nearly symmetric negative peak that has no distinct structure. The dichroic line at the $M_{5}$ edge has an asymmetric $s$ shape with two peaks - a stronger negative peak and a weaker positive peak. The overall shapes of the calculated and experimental uranium $M_{4,5}$ XMCD spectra correspond well to each other. The major discrepancy between the calculated and experimental XMCD spectra is in the amplitude of the $M_{4}$ XMCD peak. The LSDA underestimates the integral intensity of the XMCD at $M_{4}$ edge, the LSDA $+U$ approach overestimates the dichroism at this edge. The LSDA+OP approximation, in contrast, gives the best agreement with the experimental spectrum. Similar situation was observed in the case of the $M_{5}$ XMCD spectrum where the LSDA+OP gives the best agreement with the experimental spectrum.

The line shape of the dichroic spectra can be qualitatively understood considering the MO selection rules as well as the occupation and the energy sequence of the $m_{j}$-projected partial densities of states. The $5 f_{7 / 2}$ states are almost completely empty in all the uranium compounds and the XMCD spectrum of $\mathrm{U}$ at the $M_{5}$ edge can be roughly approximated by the following sum of partial densities of $5 f_{7 / 2}$ states: $\left(N_{-7 / 2}^{7 / 2}+N_{-5 / 2}^{7 / 2}\right)-\left(N_{7 / 2}^{7 / 2}+N_{5 / 2}^{7 / 2}\right)$. As a result, the shape of the $M_{5}$ XMCD spectrum stems from two peaks of opposite signs - a negative peak at lower energy and a positive peak at higher energy. As the separation of the peaks is smaller than the typical lifetime broadening, the peaks cancel each other to a large extent, thus leading to a rather small signal.
A rather different situation occurs in the case of the $M_{4}$ X-ray absorption spectrum. Uranium compounds have partially occupied $5 f_{5 / 2}$ states and the XMCD spectrum of $U$ at the $M_{4}$ edge can be approximated by $-\left(N_{3 / 2}^{5 / 2}+N_{5 / 2}^{5 / 2}\right)$. This explains why the dichroic $M_{4}$ line in uranium compounds consists of a single nearly symmetric negative peak. The XMCD signals at $\mathrm{U} M_{2,3}, N_{2,3}, O_{2,3}$ and $N_{6,7}$ edges are two orders of magnitude weaker than the corresponding signals at the $M_{4,5}$ edges.

Due to small exchange splitting of the initial $1 s$-core states only the exchange and spin-orbit splitting of the final $4 p$ states are responsible for the observed dichroism at Co and Ge $K$ edges. The XMCD spectra of Co for the $L_{2,3}$ edge are mostly determined by the strength of the SO coupling of the initial $2 p$-core states and spinpolarization of the final empty $3 d_{3 / 2,5 / 2}$ states while the exchange splitting of the $2 p$-core states as well as the SO coupling of the $3 d$ valence states are of minor importance.

The $5 f$ electron count is $2<n_{e}^{5 f}<3$ from the XMCD measurement and close to 3 from core level photoelectron spectroscopy. This is in agreement with our band structure calculations, $n_{e}^{5 f}$ is estimated to be 2.92 (in the LSDA calculations) and 2.96 (for the GGA approach). Therefore valency of $\mathrm{U}$ ion in $\mathrm{UCoGe}$ is close to $3+$. The ratio $M_{l} / M_{S}$ ranged from 1.163 in the GGA approach up to 2.456 for the LSDA $+U$ calculations is smaller than the corresponding ratio for the free ion $\mathrm{U}^{3+}$ value (2.60), it can indicate a significant delocalization of the $5 f$ electron states due to the hybridization of the $\mathrm{U} 5 f$ electrons with the conduction band and Co $3 d$ electrons.

\section{Acknowledgments}

This work was supported by Science and Technology Center in Ukraine STCU, Project No. 6255.

1. V.L. Ginzburg, Sov. Phys. JETP 4, 153 (1957).

2. N. Aso, G. Motoyama, Y. Uwatoko, S. Ban, S. Nakamura, T. Nishioka, Y. Homma, Y. Shiokawa, K. Hirota, and N.K. Sato, Phys. Rev. B 73, 054512 (2006).

3. S.S. Saxena, P. Agarwal, K. Ahilan, F.M. Grosche, R.K.W. Haselwimmer, M. Steiner, E. Pugh, I.R. Walker, S.R. Julian, P. Monthoux, G.G. Lonzarich, A. Huxley, I. Sheikin, D. Braithwaite, and J. Flouquet, Nature 406, 587 (2000).

4. D. Aoki, A. Huxley, E. Ressouche, D. Braithwaite, J. Flouquet, J.P. Brison, E. Lhotel, and C. Paulsen, Nature 413, 613 (2001).

5. N.T. Huy, A. Gasparini, D.E. de Nijs, Y. Huang, J.C.P. Klaasse, T. Gortenmulder, A. de Visser, A. Hamann, T. Gorlach, and H.V. Lohneysen, Phys. Rev. B 99, 067006 (2007).

6. D. Aoki and J. Flouquet, J. Phys. Soc. Jpn. 81, 011003 (2012) 
7. V. Sechovsky and L. Havela, in: Intermetallic Compounds of Actinides. Ferromagnetic Materials, E.P. Wohlfarth and K.H.J. Buschow (eds.), Elsvier, Amsterdam (1998), vol. 4.

8. D. Aoki, I. Sheikin, T.D. Matsuda, V. Taufour, G. Knebel, and J. Flouquet, J. Phys. Soc. Jpn. 80, 013705 (2011).

9. L. Malone, L. Howald, A. Pourret, D. Aoki, V. Taufour, G. Knebel, and J. Flouquet, Phys. Rev. B 85, 024526 (2012).

10. E. Steven, A. Kiswandhi, D. Krstovska, J.S. Brooks, M. Almeida, A.P. Goncalves, M.S. Henriques, G. M. Luke, and T.J. Williams, Appl. Phys. Lett. 98, 132507 (2011).

11. G.H. Lande, M.S.S. Brooks, and B. Johansson, Phys. Rev. B 43, 13672 (1991).

12. M. Samsel-Czekala, S. Elgazzar, P.M. Oppeneer, E. Talik, W. Walerczyk, and R. Troc, J. Phys.: Condens. Matter 22, 015503 (2010).

13. K. Karube, T. Hattori, Y. Ihara, Y. Nakai, K. Ishida, N. Tamura, K. Deguchi, N.K. Sato, and H. Harima, J. Phys. Soc. Jpn. 80, 064711 (2011).

14. K. Prokes, A. de Visser, Y.K. Huang, B. Fak, and E. Ressouche, Phys. Rev. B 81, 180407 (2010).

15. M. Taupin, J.-P. Sanchez, J.-P. Brison, D. Aoki, G. Lapertot, F. Wilhelm, and A. Rogalev, Phys. Rev. B 92, 035124 (2015).

16. M.W. Butchers, J.A. Duffy, J.W. Taylor, S.R. Giblin, S.B. Dugdale, C. Stock, P.H. Tobash, E.D. Bauer, and C.Paulsen, Phys. Rev. B 92, 121107(R) (2015).

17. F. Canepa, P. Manfrinetti, M. Pani, and A. Palenzona, J. Alloys Comp. 234, 225 (1996).

18. H.H. Hill, Plutonium and Other Actinides, W.N. Miner (ed.), AIME, New York (1970).

19. G.Y. Guo, H. Ebert, W.M. Temmerman, and P.J. Durham, Phys. Rev. B 50, 3861 (1994).

20. V.N. Antonov, A.I. Bagljuk, A.Y. Perlov, V.V. Nemoshkalenko, V.N. Antonov, O.K. Andersen, and O. Jepsen, Fiz. Niz. Temp. 19, 689 (1993) [Low Temp. Phys. 19, 494 (1993)].

21. V. Antonov, B. Harmon, and A. Yaresko, Electronic Structure and Magneto-Optical Properties of Solids, Kluwer, Dordrecht (2004).

22. E. Arola, M. Horne, P. Strange, H. Winter, Z. Szotek, and W.M. Temmerman, Phys. Rev. B 70, 235127 (2004).

23. G. van der Laan and B.T. Thole, Phys. Rev. B 38, 3158 (1988).

24. B.T. Thole, P. Carra, F. Sette, and G. van der Laan, Phys. Rev. Lett. 68, 1943 (1992).

25. P. Carra, B.T. Thole, M. Altarelli, and X. Wang, Phys. Rev. Lett. 70, 694 (1993).

26. G. van der Laan and B.T. Thole, Phys. Rev. B 53, 14458 (1996).

27. F.K. Richtmyer, S.W. Barnes, and E. Ramberg, Phys. Rev. 46, 843 (1934).

28. V.N. Antonov, O. Jepsen, A.N. Yaresko, and A.P. Shpak, J. Appl. Phys. 100, 043711 (2006).

29. V.N. Antonov, M. Galli, F. Marabelli, A.A. Yaresko, A.Ya. Perlov, and E. Bauer, Phys. Rev. B 62, 1742 (2000).

30. O.K. Andersen, Phys. Rev. B 12, 3060 (1975).
31. V.V. Nemoshkalenko, A.E. Krasovskii, V.N. Antonov, V.N. Antonov, U. Fleck, H. Wonn, and P. Ziesche, Phys. Status Solidi B 120, 283 (1983).

32. V.N. Antonov, A.Y. Perlov, A.P. Shpak, and A.N. Yaresko, J. Magn. Magn. Mater. 146, 205 (1995).

33. J. Perdew and Y. Wang, Phys. Rev. B 45, 13244 (1992).

34. J.P. Perdew, K. Burke, and M. Ernzerhof, Phys. Rev. Lett. 77, 3865 (1996).

35. J.P. Perdew, K. Burke, and M. Ernzerhof, Phys. Rev. Lett. 78, 1396 (1997).

36. P.E. Blöchl, O. Jepsen, and O.K. Andersen, Phys. Rev. B 49, 16223 (1994).

37. J. L. Campbell and T. Parr, At. Data Nucl. Data Tables 77, 1 (2001).

38. M.S.S. Brooks and B. Johansson, in: Handbook of Magnetic Materials, vol. 7, K.H.J. Buschow (ed.), North-Holland, Amsterdam (1993), p. 139

39. M.S.S. Brooks, Physica B 130, 6 (1985).

40. O. Eriksson, M.S.S. Brooks, and B. Johansson, Phys. Rev. B 41, 7311 (1990).

41. L. Severin, M.S.S. Brooks, and B. Johansson, Phys. Rev. Lett. 71, 3214 (1993).

42. A. Mavromaras, L. Sandratskii, and J. Kübler, Solid State Commun. 106, 115 (1998).

43. G. Vignale and M. Rasolt, Phys. Rev. Lett. 59, 2360 (1987).

44. P. Skudlarski and G. Vigmale, Phys. Rev. B 48, 8547 (1993).

45. M. Higuchi and A. Haegawa, J. Phys. Soc. Jpn. 66, 149 (1997).

46. S.V. Beiden, W.M. Temmerman, Z. Szotek, and G.A. Gehring, Phys. Rev. Lett. 79, 4970 (1997).

47. I.V. Solovyev, A.I. Liechtenstein, and K. Terakura, Phys. Rev. Lett. 80, 5758 (1998).

48. A.N. Yaresko, V.N. Antonov, and P. Fulde, Phys. Rev. B 67 , 155103 (2003).

49. V.I. Anisimov, J. Zaanen, and O.K. Andersen, Phys. Rev. B 44, 943 (1991).

50. M. Divis, Physica B 403, 2505 (2008).

51. J.-X. Yu, Y. Cheng, B. Zhu, and H. Yang, Physica B 406, 2788 (2011).

52. M. Valiska, J. Posps, M. Divis, J. Prokleska, V. Sechovsky, and M.M. Abd-Elmeguid, Phys. Rev. B 92, 045114 (2015).

53. S. Fujimori, T. Ohkochi, I. Kawasaki, A. Yasui, Y. Takeda, T. Okane, Y. Saitoh, A. Fujimori, H. Yamagami, Y. Haga, et al., Phys. Rev. B 91, 174503 (2015).

54. P.D. de Reotier, A. Yaouanc, G. van der Laan, N. Kernavanois, J.P. Sanchez, J.L. Smith, A. Hiess, A. Huxley, and A. Rogalev, Phys. Rev. B 60, 10606 (1999).

55. H. Ebert, J. Phys.: Condens. Matter 1, 9111 (1989).

56. H.J. Gotsis and P. Strange, J. Phys.: Condens. Matter 6, 1409 (1994).

57. M.S.S. Brooks and B. Johansson, in: Spin-Orbit Influenced Spectroscopies, H. Ebert and G. Schütz (eds.), Springer, Berlin (1996), p. 211.

58. V.N. Antonov, B.N. Harmon, and A.N. Yaresko, Phys. Rev. $B$ 63, 205112 (2001). 
59. G.G. Scott, J. Phys. Soc. Jpn. 17, 372 (1962).

60. W. Marshall and S.W. Lovsey, Theory of Thermal Neutron Scattering, Oxford University Press, Oxford (1971).

61. M. Blume, J. Appl. Phys. 57, 3615 (1985).

62. R. Troc and V.H. Tran, J. Magn. Magn. Mater. 73, 389 (1988).
63. P. Mora and O. Navarro, J. Phys.: Condens. Matter 20, 285221 (2008).

64. S. Fujimori, T. Ohkochi, I. Kawasaki, A. Yasui, Y. Takeda, T. Okane, Y. Saitoh, A. Fujimori, H. Yamagami, Y. Haga, E. Yamamoto, Y. Tokiwa, S. Ikeda, T. Sugai, H. Ohkuni, N. Kimura, and Y. Ōnuki, J. Phys. Soc. Jpn. 81, 014703 (2012). 